\title{
Ganglionated Plexi Ablation to Treat Patients with Refractory Neurally Mediated Syncope and Severe Vagal-Induced Bradycardia
}

\author{
Mauricio Scanavacca ${ }^{\circledR}$ and Denise Hachul ${ }^{\circledR}$ \\ Instituto do Coração da Faculdade de Medicina da Universidade de São Paulo, São Paulo, SP - Brazil
}

Neurally mediated syndrome (NMS) is the most common cause of and transient loss of consciousness and usually causes anxiety and concerns for patients and their families. Clinical investigation and explanations about benignity, in association to lifestyle modifications and teaching methods to abort the vagal reflex are the initial and effective approaches to manage the majority of patients. ${ }^{1-3}$

However, in some cases, the loss of consciousness occurs suddenly, without prodromal symptoms and the patient does not have the opportunity to prevent falls, which may lead to severe physical trauma. Specific medications have been proposed trying to control the symptoms and pacemaker implantation has been recommended in the refractory cases of cardioinhibitory reflexes. ${ }^{1-3}$

In 2005, Pachón et al. ${ }^{4}$ proposed using a catheter ablation technique to attenuate vagal activity on the sinus and AV nodes, through radiofrequency (RF) ablation of the sites related to vagal inputs to the atria. This procedure aimed to promote better quality of live for patients with refractory NMS and severe bradycardia, avoiding pacemaker implantation, especially in young individuals. ${ }^{4}$ Since that time, many reports have confirmed that atrial vagal modulation by catheter ablation is feasible in clinical settings, for patients with vagal induced marked and symptomatic bradycardia. ${ }^{5-20}$

However, those studies came from relatively few centers, such as case reports, ${ }^{5-13}$ or from non-randomized series involving a limited number of patients. ${ }^{14-20}$ Additionally, until now, there is no consensus about the criteria for patient selection, about how to perform an autonomic evaluation prior and post ablation, the best technique to be applied, the endpoints to conclude the procedure and what to expect during the outcome. These are the main reasons why vagal attenuation by catheter ablation has not been considered a possible treatment in the international guidelines of NMS management. ${ }^{1-3}$

Almost 50 years ago, experimental studies demonstrated that the sinus and atrioventricular nodes receive specific autonomic innervation, which can be surgically destroyed. ${ }^{21}$ However, current techniques to promote vagal denervation have been based on a more recent descriptions of atria innervation. ${ }^{22,23}$ It has been demonstrated that cardiac parasympathetic neurons get together with postganglionic

\section{Keywords}

Ganglia, Autonomic; Catheter Ablation; Syncope, Vasovagal; Bradycardia; Autonomic Nervous System.

Mailing Address: Mauricio Ibrahim Scanavacca •

Av. Joaquim C. A. Marques, 1205. Postal Code 05688-021, Morumbi, SP - Brazil E-mail: mibrahim@cardiol.br, mauricio.scanavacca@gmail.com

DOI: $10.5935 / a b c .20190107$ sympathetic fibers and fat tissue (fat pads), in specific areas of the epicardium, adjacent to the posterior walls of left and right atria. Such ganglionated plexi (GP), working together, coordinate a sophisticated network that regulates cardiac functions, and more precisely the cardiac rhythm. ${ }^{24}$

The Oklahoma group has worked intensively to understand GP functional activities and has observed, in experimental studies, that despite the intense interconnections among GP, the sinus node is mainly innervated by the plexi, anatomically situated in the superior portion of the posterior interatrial septum, between the superior vena cava and anterior to the right superior PV. The AV node is manly innervated by the right inferior plexi, located behind the coronary sinus ostium and between the inferior vena cava and right inferior PV. The sinus node and AV node still receive fiber connections from the left superior and left inferior GP located nearby the left pulmonary veins. However, these interconnections necessary cross the right superior and right inferior PV GP to reach the sinus and AV nodes. ${ }^{24}$ Additionally, extra GPs connections might influence the sinus and AV node innervation. ${ }^{22-24}$ It has also been suggested that the intrinsic autonomic nervous system of the heart receives inputs from mechanical and chemosensory receptors located in both ventricles. ${ }^{25}$ Those autonomic nervous interconnections could have an important role in the pathophysiology of NMS and might explain some behaviors observed in patients submitted to GPs ablation, who showed additional improvement in the peripheral vasovagal reflex.

Sinus node vagal denervation was a fortuitous observation detected by the occurrence of an augmented vagal response triggered during catheter ablation of atrial fibrillation and also, the subsequent increase in heart rate observed soon after the procedure. Those findings were more evident when extensive ablation of the PV antrum, where GPs are frequently located, was performed. ${ }^{26,27}$ Autonomic tests performed after PV isolation confirmed an effect of the vagal denervation of the sinus node in many patients; however, a controversy still persists regarding the effect of persistent atrial vagal denervation on the long term outcome. ${ }^{28,29}$ Those serendipity findings opened a new possibility for the treatment of the negative effects of excessive vagal activity in some patients.

Different techniques have been used for sinus and AV node vagal denervation: one is based on the hypothesis that autonomic innervation can be recognized by the characteristics of the endocardial fractionated electrograms (AF Nests), and detected by Fast-Fourier transform analysis. ${ }^{4}$ The original report from Pachón et al. ${ }^{5}$ in 2005, described twenty-one patients with a mean age of 48 years, six with neurally mediated reflex syncope, and 15 with sinus node dysfunction or functional high-degree atrioventricular block, who underwent vagal denervation. In a mean follow-up 
of 9.2 months, no patient showed syncope recurrence, or complications. In 2011, the authors reported the late outcome of 43 patients with recurrent NMS, submitted to ablation. Forty patients remained asymptomatic during a mean follow-up of $45.1 \pm 22$ months and no significant complications were observed. ${ }^{5}$

More recently, Yan et al. ${ }^{14}$ performed vagal denervation in ten consecutive patients with a mean age of $50.4 \pm 6.4$ years and recurrent episodes of NMS, in which traditional therapies were not effective. The authors used the high-frequency stimulation (HFS) technique, described by the Oklahoma group, in order to locate GP based on induced vagal response. ${ }^{30}$ The endpoint of the procedure was the inhibition of the vagal response at the target sites. During a mean follow-up of $30 \pm 16$ months, no patient had syncope episodes, but 5 patients reported transient symptoms. There were no complications. ${ }^{14}$

Sun et al. ${ }^{15}$ reported the long-term outcome of 57 consecutive patients (aged $43.2 \pm 13.4$ years; 35 women) with refractory vasovagal syncope, who underwent left atrium GP ablation. The GP were located based on the combination of anatomic features obtained by the electroanatomic mapping and positive response (vagal hyperactivity) during HFS, on expected GP locations. During a mean follow-up of $36 \pm 22 \mathrm{~m}$, $52(91.2 \%)$ patients had no syncope. However, 16 patients showed prodromal symptoms. Autonomic evaluation revealed reduced vagal activity, persistent for at least 12 months after the procedure. The only side effect was sinus tachycardia observed in one patient. ${ }^{15}$ Zhao et al. ${ }^{16}$ performed catheter ablation in 11 patients ( 8 men, with mean age of $45 \pm 10$ years) with a long history of symptomatic bradycardia. All anatomic locations of the GP were confirmed by HF stimulation before ablation. The procedure endpoint was the elimination of vagal response at the ablation sites. During a mean follow-up of $18 \pm 6$ months, all patients reported significant symptom improvement. There was a significantly increase in the mean sinus heart rate, which persisted for 12 months. ${ }^{16}$ The main limitations of these studies are the absence of control groups and randomization. The already known placebo effect of interventions in patients with NMS does not allow considering their good results as definitive to regularly apply the GP ablation in clinical practice.

Despite such limitations many other authors have reported selected cases in which patients had advanced atrioventricular block induced by excessive vagal activity and had indication for pacemaker impantation. ${ }^{6-13}$ Although the GP mapping and ablation technique were not identical, no patient had complications and pacemaker implantation was avoided. Some authors delivered RF pulses guided by a positive vagal action induced by HFS. Others delivered RF pulses based on the characteristics of the electrograms, or at the anatomic sites where the presence of GP clusters was highly probable, based on previous anatomical reports. ${ }^{17-20}$

We investigated the effectiveness of vagal ablation in 14 patients ( 7 men, mean age of $34 \pm 13$ years) with symptomatic cardioinhibitory syncope, severe sinus bradycardia or advanced AV block, no structural heart disease, and pacemaker indication. The GP were identified and ablated based on the anatomy obtained by the electroanatomical mapping analysis. During a mean follow-up period of $22 \pm 11$ months, 10 patients (71.4\%) showed significant clinical and ECG improvement: no syncope recurrence or symptomatic bradycardia were observed at daytime Holter monitoring during the follow-up. However, transient second-degree atrioventricular block was still detected, exclusively at night. The remaining four patients (28.6\%), despite showing acute vagal activity attenuation, had syncope recurrence or symptomatic bradycardia and underwent pacemaker implantation. No significant complications were observed during or after the procedure. ${ }^{18}$

Therefore, until now, no consensus has been achieved about the ideal electrophysiological technique to perform vagal activity attenuation: if through extensive ablation areas based on fragmented electrograms, or by anatomical landmarks; if ablating all right and left atrial GP areas, or those that directly innerve the sinus and AV nodes, in both sides of the septum; or only the right side of the septum, or even spots in the superior vena cava. ${ }^{9,10,20}$ Another important controversial point is how to establish the procedure endpoint, whether reaching an expected heart rate elevation such as $20 \%$ of basal cardiac frequency, shortening the P-P interval > 120 ms; eliminating prior vagal response induced by HFS on the target GP or absence of an additional heart rate elevation after GP ablation with the atropine test. Recently, an extracardiac vagal stimulation with high frequency pulses, performed directly on the right or left cervical vagal trunk through retrograde jugular vein catheterization was suggested to evaluate vagal response before, during and the end of the procedure. This method has been proposed to obtain an objective, faster and reproducible evaluation of the ablation effect during the procedure. ${ }^{31}$

Finally, it is important to define a reproducible autonomic evaluation in patients considered for vagal denervation. The most frequent analysis prior to the procedure has been the analysis of time-domain and frequency-domain heart rate variability (HRV), during $24 \mathrm{~h}$ Holter monitoring. Small segments of the ECG are also evaluated during the tilt table test, in the last 5 minutes of supine position and the first 5 minutes of orthostatic exposure. It is also important to identify patients with the vasodepressor component, in which prodromal symptoms will probably persist, even after effective sinus and/or AV node denervation. The atropine test $(0.04 \mathrm{mg} / \mathrm{Kg})$, complemented with exercise stress test, have been performed to evaluate the vagal reserve and to rule out a possible sinus node dysfunction. Such autonomic evaluation should be repeated during the follow-up aiming to identify the long-term outcome of the autonomic modulation.

In summary, the experimental and clinical background suggest that RF ablation of GP may promote significant vagal withdrawal in the sinus and AV nodes, in patients with very symptomatic neurally mediated bradycardia syndromes. However, its effectiveness, safety and reproducibility need a more thorough evaluation. Different techniques have been proposed to successfully identify and ablate the sites of vagal inputs to the atria. Therefore, a randomized multicenter study is fundamental not just to establish the effectiveness of vagal denervation and the long-term clinical impact on symptoms and safety to the patient but also to identify the best techniques to obtain vagal response attenuation, with smaller areas of ablation. 


\section{References}

1. Sheldon RS, Grubb BP, Olshansky B Shen WK, Calkins H, Brignole M, et al. et al. 2015 Heart Rhythm Society expert consensus statement on the diagnosis and treatment of postural tachycardia syndrome, inappropriate sinus tachycardia, and vasovagal syncope. Heart Rhythm. 2015; 12(6):e41-63.

2. Shen WK, Sheldon RS, Benditt DG, Cohen MI, Forman DE, Goldberger ZD, et al. 2017 ACC/AHA/HRS guideline for the evaluation and management of patients with syncope: A report of the American College of Cardiology/ American Heart Association Task Force on Clinical Practice Guidelines and the Heart Rhythm Society. Heart Rhythm. 2017; 14(8):e155-e217.

3. Brignole M, Moya A, de Lange FJ, Deharo JC, Elliott, Fancielli A, et al. 2018 ESC Guidelines for the diagnosis and management of syncope. Eur Heart J. 2018; 39(21):1883-948.

4. Pachón JC, Pachón El, Pachón JC, Lobo TJ, Pachon MZ, Vargas RN, et al. "Cardioneuroablation" - New treatment for neurocardiogenic syncope, functional AV block and sinus dysfunction using catheter RF-ablation. Europace. $2005 ; 7(1): 1-13$.

5. Pachón JC, Pachón El, Cunha Pachón MZ, Lobo TJ, Pachon JC, Santillana TG et al. Catheter ablation of severe neurally meditated reflex (neurocardiogenic or vasovagal) syncope: cardioneuroablation long-term results. Europace. $2011 ; 13(9): 1231-42$

6. Pachón MJC, Pachón M EI, Lobo TJ,Pachon MC, Pachon MZ, Vargas RN, et al. Syncopal high- degree AV block treated with catheter RF ablation without pacemaker implantation. Pacing Clin Electrophysiol. 2006; 29(3):318-22.

7. Scanavacca M, Hachul D, Pisani C, Sosa E. Selective vagal denervation of the sinus and atrioventricular nodes, guided by vagal reflexes induced by high frequency stimulation, to treat refractory neurally mediated syncope. J Cardiovasc Electrophysiol. 2009;20(5):558-63.

8. Liang Z, Jiayou Z, Zonggui W, Dening L. Selective atrial vagal denervation guided by evoked vagal reflex to treat refractory vasovagal syncope. Pacing Clin Electrophysiol. 2012;35(7):214-8.

9. Rebecchi M, de Ruvo E, Strano S, Sciarra L, Golia P, Martino A, et al. Ganglionated plexi ablation in right atrium to treat cardioinhibitory neurocardiogenic syncope. J Interv Card Electrophysiol. 2012;34(3):231-5.

10. Suenaga H, Murakami M, Tani T, Saito S. Frequent neurally mediated reflex syncope in a young patient with dextrocardia: Efficacy of catheter ablation of the superior vena cava-aorta ganglionated plexus. J Arrhythm. 2015;31(3):172-6.

11. Aksu T, Golcuk SE, Guler TE, Yalin K, Erden J. Functional permanent 2:1 atrioventricular block treated with cardioneuroablation: Case report. Heart Rhythm Case Rep. 2015;1(2):58-61.

12. Fukunaga M, Wichterle D, Peichl P, Aidhoon B, Cihak R, Kautzner J, et al. Differential effect of ganglionic plexi ablation in a patient with neurally mediated syncope and intermittent atrioventricular block. Europace. 2016. pii:euw100.

13. Rivarola E, Hardy C, Sosa E, Hachul D, Furlan V, Raimundi F, et al. Selective atrial vagal denervation guided by spectral mapping to treat advanced atrioventricular block. Europace. 2016;18(3):445-9.

14. Yao Y, Shi R, Wong T,Chen W, Yang L, et al. Endocardial autonomic denervation of the left atrium to treat vasovagal syncope: an early experience in humans. Circ Arrhythm Electrophysiol. 2012;5(2):279-86.

15. Sun W, Zheng L, Qiao Y, Shi R, Hou B, Wu L, et al. Catheter ablation as a treatment for vasovagal syncope: long-term outcome of endocardial autonomic modification of the left atrium. J Am Heart Assoc. 2016;5(7):pii e003471.
16. Zhao L, Jiang W, Zhou L, Wang Y, Zhang X, Xu K, et al. Atrial autonomic denervation for the treatment of long-standing symptomatic sinus bradycardia in non-elderly patients. J Interv Card Electrophysiol. 2015;43(2):151-9.

17. Aksu T, Golcuk E, Yalin K, Guler TE, Erden I. Simplified cardioneuroablation in neurally mediated reflex syncope. Pacing Clin Electrophysiol 2016;39(1):42-53.

18. Rivarola E, Hachul D, Wu T, Pisani C, Hardy C, Raimundi F, et al. Targets and endpoints in cardiac autonomic denervation procedures. Circ Arrhythm Electrophysiol. 2017;10(2):e004638.

19. Qin M, Zhang Y, Liu X, Jiang WF, Wu SH, Atrial ganglionated plexus modification: a novel approach to treat symptomatic sinus bradycardia J Am Coll Cardiol.2017(9):950-9.

20. Debruyne P, Rossenbacker T, Collienne C. Unifocal right-sided ablation treatment for neurally mediated syncope and functional sinus node dysfunction under computed tomographic guidance. Circ Arrhythm Electrophysiol. 2018;11(9):e006604.

21. Randall WC, Ardell JL. Selective parasympathectomy of automatic and conductible tissues of the canine heart. Am J Physiol. 1985;248(1 Pt2):H61-8.

22. Armour JA, Murphy DA, Yuan BX. Gross and microscopic anatomy of the human intrinsic cardiac nervous system. Anat Rec. 1997;247(2):289-98.

23. Pauza DH, Skripka V, Pauziene N, Stropus R. Morphology, distribution, and variability of the epicardic neural ganglionated subplexuses in the human heart. Anat Rec. 2000;259(4):353-82.

24. Hou Y, Scherlag BJ, Lin J, Zhou J, Song J, Zhang Y, et al. Interactive atrial neural network: Determining the connections between ganglionated plexi. Heart Rhythm. 2007;4(1):56-63.

25. Thompson GW, Collier K, Ardell JL. Functional interdependence of neurons in a single canine intrinsic cardiac ganglionated plexus. J Physiol. 2000;528(1):561-71.

26. Pappone C, Santinelli V, Manguso F, Vicedomini G, Gugliotta F, Augello $\mathrm{G}$, et al. Pulmonary vein denervation enhances long-term benefit after circumferential ablation for paroxysmal atrial fibrillation. Circulation. 2004;109(3):327-34.

27. Scanavacca M, Pisani CF, Hachul D, Lara S, Hardy C, Darrieux F, et al. Selective atrial vagal denervation guided by evoked vagal reflex to treat patients with paroxysmal atrial fibrillation. Circulation. 2006;114(9):876-85.

28. Katritsis DG, Pokushalov E, Romanov A, Glazitzoglou E, Siontis GC, Po $\mathrm{SS}$. et al. Autonomic denervation added to pulmonary vein isolation for paroxysmal atrial fibrillation: a randomized clinical trial. J Am Coll Cardiol. 2013;62(24):2318-25.

29. Sakamoto S, Schuessler RB, Lee AM, Aziz A, Lall SC, Damiano R J Jr. Vagal denervation and reinnervation after ablation of ganglionated plexi. J Thorac Cardiovasc Surg. 2010;139(2):444-52.

30. Scherlag BJ, Nakagawa H, Jackman WM, Yamaneshi WS, Patterson E, Po SS, et al. Electrical stimulation to identify neural elements on the heart: their role in atrial fibrillation. J Interv Card Electrophysiol. 2005;13(Suppl 1):37-42.

31. Pachón MJC, Pachón MEI, Santillana PTG, Lobo TJ, Pachon CTC, Pachon MJ, et al. Simplified Method for Vagal Effect Evaluation in Cardiac Ablation and Electrophysiological Procedures. JACC Clin Electrophysiol. 2015;1(5):451-60. 
